\title{
Secure Remote Authentication Using Biometric Data
}

\author{
Xavier Boyen ${ }^{1}$, Yevgeniy Dodis ${ }^{2, \star}$, Jonathan Katz ${ }^{3, \star \star}$, \\ Rafail Ostrovsky ${ }^{4, \star \star \star}$, and Adam Smith ${ }^{5}$ \\ 1 Voltage, Inc. \\ xb@boyen.org \\ 2 New York University \\ dodis@cs.nyu.edu \\ 3 University of Maryland \\ jkatz@cs.umd.edu \\ 4 UCLA \\ rafail@cs.ucla.edu \\ 5 Weizmann Institute \\ asmith@csail.mit.edu
}

\begin{abstract}
Biometric data offer a potential source of high-entropy, secret information that can be used in cryptographic protocols provided two issues are addressed: (1) biometric data are not uniformly distributed; and (2) they are not exactly reproducible. Recent work, most notably that of Dodis, Reyzin, and Smith, has shown how these obstacles may be overcome by allowing some auxiliary public information to be reliably sent from a server to the human user. Subsequent work of Boyen has shown how to extend these techniques, in the random oracle model, to enable unidirectional authentication from the user to the server without the assumption of a reliable communication channel.

We show two efficient techniques enabling the use of biometric data to achieve mutual authentication or authenticated key exchange over a completely insecure (i.e., adversarially controlled) channel. In addition to achieving stronger security guarantees than the work of Boyen, we improve upon his solution in a number of other respects: we tolerate a broader class of errors and, in one case, improve upon the parameters of his solution and give a proof of security in the standard model.
\end{abstract}

\section{Using Biometric Data for Secure Authentication}

Biometric data, as a potential source of high-entropy, secret information, have been suggested as a way to enable strong, cryptographically-secure authentica-

* Supported by NSF CAREER award 0133806 and Trusted Computing grant 0311095

** Supported by NSF CAREER award 0447075 and Trusted Computing grants 0310751 and 0310499.

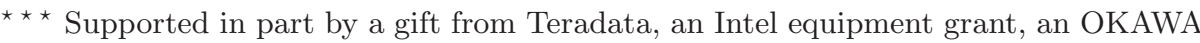
research award, and an NSF Cybertrust grant. 
tion of human users without requiring them to remember or store traditional cryptographic keys. Before such data can be used in existing cryptographic protocols, however, two issues must be addressed: first, biometric data are not uniformly distributed and hence do not offer provable security guarantees if used as is, say, as a key for a pseudorandom function. While the problem of nonuniformity can be addressed using a hash function, viewed either as a random oracle 22 or a strong extractor 20, a second and more difficult problem is that biometric data are not exactly reproducible, as two biometric scans of the same feature are rarely identical. Thus, traditional protocols will not even guarantee correctness when the parties use a shared secret derived from biometric data.

Much work has focused on addressing these problems in efforts to develop secure techniques for biometric authentication [8, 15, 19, 14, 22, 21. Most recently, Dodis, Reyzin, and Smith 9 showed how to use biometric data to securely derive cryptographic keys which could then be used, in particular, for the purposes of authentication. Roughly speaking (see Section 2 for formal definitions), they introduce two primitives: a secure sketch which allows recovery of a shared secret given a close approximation thereof, and a fuzzy extractor which extracts a uniformly distributed string $s$ from this shared secret in an error-tolerant manner. Both primitives work by constructing a "public" string pub which is stored by the server and transmitted to the user; loosely speaking, pub encodes the redundancy needed for error-tolerant reconstruction. The primitives are designed so as to be "secure" even when an adversary learns the value of this public string.

Unfortunately, although these primitives suffice to obtain security in the presence of an eavesdropping adversary who learns pub as it is sent to the user, the work of Dodis et al. does not address the issue of malicious modification of pub. As a consequence, their work does not provide a method for secure authentication in the presence of an active adversary who may modify the messages sent between the server and the user. Indeed, depending on the specific sketch or fuzzy extractor being utilized, an adversary who maliciously alters the public string sent to a user may be able to learn that user's biometric data in its entirety. A "solution" is for the user to store pub himself rather than obtain it from the server (or to authenticate pub using a certificate chain), but this defeats the purpose of using biometric data in the first place: namely, to avoid the need for the user to store any additional cryptographic information - even if that information need not be kept secret.

Boyen [5, inter alia, partially addresses potential adversarial modification of pub (although his work focuses primarily on the orthogonal issue of re-using biometric data with multiple servers, which we do not explicitly address here). The main drawback of his technique in our context is that it provides only unidirectional authentication from the user to the server. Indeed, Boyen's approach cannot be used to achieve authentication of the server to the user since his definition of "insider security" (cf. [5-Section 5.2]) does not preclude an adversary from knowing the (incorrect) value $s^{\prime}$ of the shared secret recovered by the user when the adversary forwards a specially crafted pub' to this user; if the adversary knows $s^{\prime}$, then from the viewpoint of the user the adversary can do anything the 
server could do, and hence authentication of the server to the user is impossible. The lack of mutual authentication implies that — when communicating over an insecure network - the user and server cannot securely establish a shared session key with which to encrypt and authenticate future messages: the user may unwittingly share a key with an adversary who can then decrypt any data sent by that user as well as authenticate arbitrary data.

\subsection{Our Contributions}

In this paper, we provide the first full solution to the problem of secure remote authentication using biometric datd: in particular, we show how to achieve mutual authentication and/or authenticated key exchange over a completely insecure channel. We offer two constructions. The first one is a generic solution which protects against modification of the public value pub in any context in which secure sketches or fuzzy extractors are used; thus, this solution serves as a drop-in replacement that "compiles" any protocol which is secure when pub is assumed to be transmitted reliably into one which is secure even when pub might be tampered with (we do not formalize this notion of "compilation", but rather view it as an intuitive way to understand our results). Our second construction is specific to the settings of remote authentication and key exchange, where it offers some improvements to the generic solution.

Compared with the work of Boyen [5], which was mostly concerned with the re-usability of biometrics, our constructions enjoy the following key advantages:

- Both of our solutions tolerate a stronger class of errors. In particular, Boyen's work only allows for data-independent errors, whereas our analysis handles arbitrary (but bounded) errors. We remark that small yet data-dependent errors seem natural in the context of biometric data.

- Our second solution is proven secure in the standard model.

- Our second solution achieves improved bounds on the entropy loss, on the order of 128 bits of entropy for practical choices of the parameters. This point is particularly important since the entropy of certain biometric features is roughly this order of magnitude (e.g., 173-250 bits for an iris scan [8, 13]).

Organization. We review some basic definitions as well as the sketches/fuzzy extractors of Dodis et al. 9] in Section 2, In Section 3 we introduce the notion of robust sketches/fuzzy extractors which are resilient to modification of the public value, and can be used as a generic replacement for sketches/fuzzy extractors in any application. Our second solution, which is specific to the problem of using biometric data for authentication and offers some advantages with respect to our generic construction, is described in Section 4 .

\footnotetext{
${ }^{1}$ Of course, our techniques are applicable to any scenario which relies on secret data
} that, like biometric data, are non-uniform and/or not exactly reproducible. 


\section{Definitions}

Unless explicitly stated otherwise, all logarithms are base 2 . We let $U_{\ell}$ denote the uniform distribution over $\ell$-bit strings. A metric space $(\mathcal{M}, d)$ is a finite set $\mathcal{M}$ equipped with a symmetric distance function $d: \mathcal{M} \times \mathcal{M} \rightarrow \mathbb{Z}^{+} \cup\{0\}$ satisfying the triangle inequality and such that $d(x, y)=0 \Leftrightarrow x=y$. (All metric spaces considered in this work are discrete, and the distances integer-valued.) For our application, we assume that the format of the biometric data is such that it forms a metric space under some appropriate distance function. We will not need to specify any particular metric space in our work, as our results build in a generic way on earlier sketch and fuzzy extractor constructions over any such space (e.g., those constructed in [9] for a variety of metrics).

A probability space $(\Omega, P)$ is a finite set $\Omega$ and a function $P: \Omega \rightarrow[0,1]$ such that $\sum_{\omega \in \Omega} P(\omega)=1$. A random variable $W$ defined over the probability space $(\Omega, P)$ and taking values in a set $\mathcal{M}$ is a function $W: \Omega \rightarrow \mathcal{M}$. If $(\Omega, P)$ is a probability space over which two random variables $W$ and $W^{\prime}$ taking values in a metric space $(\mathcal{M}, d)$ are defined, then we say that $d\left(W, W^{\prime}\right) \leq t$ if for all $\omega \in \Omega$ it holds that $d\left(W(\omega), W^{\prime}(\omega)\right) \leq t$.

Given a metric space $(\mathcal{M}, d)$ and a point $x \in \mathcal{M}$ we define

$$
\operatorname{Vol}_{t}^{\mathcal{M}}(x) \stackrel{\text { def }}{=}\left|\left\{x^{\prime} \in \mathcal{M} \mid d\left(x, x^{\prime}\right) \leq t\right\}\right|, \quad \operatorname{Vol}_{t}^{\mathcal{M}} \stackrel{\text { def }}{=} \max _{x \in \mathcal{M}}\left\{\operatorname{Vol}_{t}^{\mathcal{M}}(x)\right\} .
$$

The latter is the maximum number of points in any "ball" of radius $t$ in $(\mathcal{M}, d)$.

Following [9], for a pair of random variables $A$ and $B$, we define the minentropy $H_{\infty}(A)$ of $A$, and the average min-entropy of $A$ given $B$, as

$$
H_{\infty}(A)=-\log \left(\max _{a} \operatorname{Pr}[A=a]\right), \quad \bar{H}_{\infty}(A \mid B) \stackrel{\text { def }}{=}-\log \left(\operatorname{Exp}_{b \leftarrow B}\left[2^{-H_{\infty}(A \mid B=b)}\right]\right) .
$$

The statistical difference between random variables $A$ and $B$ taking values in the same set $D$ is defined as $\operatorname{SD}(A, B) \stackrel{\text { def }}{=} \frac{1}{2} \sum_{d \in D}|\operatorname{Pr}[A=d]-\operatorname{Pr}[B=d]|$.

\subsection{Secure Sketches and Fuzzy Extractors}

We review the definitions from [9] using slightly different terminology. Recall from the introduction that a secure sketch provides a way to recover a shared secret $w$ from any value $w^{\prime}$ which is a "close" approximation of $w$. More formally:

Definition 1. An $\left(m, m^{\prime}, t\right)$-secure sketch over a metric space $(\mathcal{M}, d)$ comprises a sketching procedure SS: $\mathcal{M} \rightarrow\{0,1\}^{*}$ and a recovery procedure Rec, where:

(Security) For all random variables $W$ over $\mathcal{M}$ such that $H_{\infty}(W) \geq m$, we have $\bar{H}_{\infty}(W \mid \mathrm{SS}(W)) \geq m^{\prime}$.

(Error tolerance) For all pairs of points $w, w^{\prime} \in \mathcal{M}$ with $d\left(w, w^{\prime}\right) \leq t$, it holds that $\operatorname{Rec}\left(w^{\prime}, \operatorname{SS}(w)\right)=w$.

While secure sketches address the issue of error correction, they do not address the issue of the possible non-uniformity of $W$. Fuzzy extractors, defined next, correct for this. 
Definition 2. An $(m, \ell, t, \delta)$-fuzzy extractor over a metric space $(\mathcal{M}, d)$ comprises a (randomized) extraction algorithm Ext : $\mathcal{M} \rightarrow\{0,1\}^{\ell} \times\{0,1\}^{*}$ and a recovery procedure Rec such that:

(Security) For all random variables $W$ over $\mathcal{M}$ that satisfy $H_{\infty}(W) \geq m$, if $\langle R, \mathrm{pub}\rangle \leftarrow \operatorname{Ext}(W)$ then $\mathbf{S D}\left(\langle R, \mathrm{pub}\rangle,\left\langle U_{\ell}, \mathrm{pub}\right\rangle\right) \leq \delta$.

(Error tolerance) For all pairs of points $w, w^{\prime} \in \mathcal{M}$ with $d\left(w, w^{\prime}\right) \leq t$, if $\langle R, \operatorname{pub}\rangle \leftarrow \operatorname{Ext}(w)$ then it is the case that $\operatorname{Rec}\left(w^{\prime}, \mathrm{pub}\right)=R$.

As shown in [9-Lemma 3.1], it is easy to construct a fuzzy extractor over a metric space $(\mathcal{M}, d)$ given any secure sketch defined over the same space, by applying a strong extractor [20 using a random "key" which is then included as part of pub. Starting with an $\left(m, m^{\prime}, t\right)$-secure sketch and with an appropriate choice of extractor, this transformation yields an $\left(m, m^{\prime}-2 \log \left(\frac{1}{\delta}\right), t, \delta\right)$-fuzzy extractor.

\subsection{Modeling Error in Biometric Applications}

As error correction is a key motivation for our work, it is necessary to develop some formal model of the types of errors that may occur. In prior work by Boyen 5, the error in various biometric readings was assumed to be under adversarial control, with the restriction that the adversary could only specify data-independent errors (e.g., constant shifts, permutations, etc.). It is not clear that this is a realistic model in practice, as one certainly expects, say, portions of the biometric data where "features" are present to be more susceptible to error.

Here, we consider a much more general error model where the errors may be data-dependent and hence correlated not only with each other but also with the biometric secret itself. Furthermore, as we are ultimately interested in modeling "nature" - as manifested in the physical processes that cause fluctuations in the biometric measurements — we do not even require that the errors be efficiently computable (although we will impose this requirement in Section 4). The only restriction we make is that the errors be "small" and, in particular, less than the desired error-correction bound; since the error-correction bound in any realworld application should be selected to ensure correctness with high probability, this restriction seems reasonable. Formally:

Definition 3. $A$ t-bounded distortion ensemble $\mathcal{W}=\left\{W_{i}\right\}_{i=0, \ldots}$ is a sequence of random variables $W_{i}: \Omega \rightarrow \mathcal{M}$ such that for all $i$ we have $d\left(W_{0}, W_{i}\right) \leq t$. $\diamond$

For our application, $W_{0}$ represents the biometric reading obtained when a user initially registers with a server, and $W_{i}$ represents the biometric reading on the $i^{\text {th }}$ authentication attempt by this user. Note that, regardless of the protocol used, an adversary can always impersonate the server if the adversary can guess $W_{i}$ for some $i>0$. The following lemmas give bound the probability of this occurrence. First, we show that the min-entropy of each $W_{i}$ is, at worst, $\log \left(\mathrm{Vol}_{t}^{\mathcal{M}}\right)$ bits less than that of $W_{0}$. Moreover, we show that $W_{i}$ is no easier to guess than $W_{0}$ when $\mathrm{SS}\left(W_{0}\right)$ is available. 
Lemma 1. Let $W_{0}, W_{1}$ be random variables over $\mathcal{M}$ satisfying $d\left(W_{0}, W_{1}\right) \leq t$, and let $B$ be an arbitrary random variable. Then

$$
\bar{H}_{\infty}\left(W_{1} \mid B\right) \geq \bar{H}_{\infty}\left(W_{0} \mid B\right)-\log \operatorname{Vol}_{t}^{\mathcal{M}} .
$$

Proof. Fix $x \in \mathcal{M}$ and any outcome $B=b$. Since $d\left(W_{0}, W_{1}\right) \leq t$, we have $\operatorname{Pr}\left[W_{1}=x \mid B=b\right] \leq \sum_{x^{\prime} \mid d\left(x, x^{\prime}\right) \leq t} \operatorname{Pr}\left[W_{0}=x^{\prime} \mid B=b\right] \leq \operatorname{Vol}_{t}^{\mathcal{M}} \cdot 2^{-H_{\infty}\left(W_{0} \mid B=b\right)}$, which means that $H_{\infty}\left(W_{1} \mid B=b\right) \geq H_{\infty}\left(W_{0} \mid B=b\right)-\log \operatorname{Vol}_{t}^{\mathcal{M}}$. Since this relation holds for every $b$, the lemma follows.

Secure sketches imply the following, stronger form of Lemma 1 which essentially states that points close to $W_{0}$ cannot be easier to guess than $W_{0}$ if the value of the sketch $\mathrm{SS}\left(W_{0}\right)$ is known.

Lemma 2. Let $W_{0}, W_{1}$ be random variables over $\mathcal{M}$ satisfying $d\left(W_{0}, W_{1}\right) \leq t$, and let $B$ be an arbitrary random variable. Let (SS, Rec) be $a(\star, \star, t)$-secure sketch. Then

$$
\bar{H}_{\infty}\left(W_{1} \mid \mathrm{SS}\left(W_{0}\right), B\right) \geq \bar{H}_{\infty}\left(W_{0} \mid \mathrm{SS}\left(W_{0}\right), B\right) .
$$

Proof. Notice that since $d\left(W_{0}, W_{1}\right) \leq t$, we have $\operatorname{Rec}\left(W_{1}, \mathrm{SS}\left(W_{0}\right)\right)=W_{0}$, which means that if for some $x, b$, pub we have $\operatorname{Pr}\left(W_{1}=x \mid \mathrm{SS}\left(W_{0}\right)=\right.$ pub, $\left.B=b\right) \geq \alpha$, then $\operatorname{Pr}\left(W_{0}=\operatorname{Rec}(x\right.$, pub $) \mid \mathrm{SS}\left(W_{0}\right)=$ pub, $\left.B=b\right) \geq \alpha$ as well. Since this holds for all $x, b$ and pub, the lemma follows.

The analogue of Lemma 2 for fuzzy extractors holds as well (with $\mathrm{SS}\left(W_{0}\right)$ replaced by pub).

\section{$3 \quad$ Robust Sketches and Fuzzy Extractors}

Recall that a secure sketch, informally speaking, takes a secret $w$ and returns some value pub which allows the recovery of $w$ given any "close" approximation $w^{\prime}$ of $w$. When pub is transmitted to a user over an insecure network, however, an adversary might modify pub in transit. In this section, we define the notion of a robust sketch which protects against this sort of attack in a very strong way: with high probability, the user will detect that pub has been modified and can thus immediately abort in this case. A robust fuzzy extractor is defined similarly. We then show: (1) a construction of a robust sketch in the random oracle model, starting from any secure sketch; and (2) a conversion from any robust sketch to a robust fuzzy extractor; this conversion does not require random oracles. We conclude this section by showing the immediate application of robust fuzzy extractors to the problem of mutual authentication.

We first define a slightly stronger notion of a secure sketch:

Definition 4. An ( $\left.m, m^{\prime}, t\right)$-secure sketch (SS, Rec) is said to be well-formed if it satisfies the conditions of Definition 1 except for the following modifications: (1) Rec may now return either an element in $\mathcal{M}$ or the distinguished symbol $\perp$; and (2) for all $w^{\prime} \in \mathcal{M}$ and arbitrary $\operatorname{pub}^{\prime}$, if $\operatorname{Rec}\left(w^{\prime}, \operatorname{pub}^{\prime}\right) \neq \perp$ then $d\left(w^{\prime}, \operatorname{Rec}\left(w^{\prime}, \operatorname{pub}^{\prime}\right)\right) \leq t$. 
It is straightforward to transform any secure sketch (SS, Rec) into a well-formed secure sketch (SS, $\operatorname{Rec}^{\prime}$ ): $\operatorname{Rec}^{\prime}$ runs $\operatorname{Rec}$ and then verifies that its output $w$ is within distance $t$ of the input $w^{\prime}$. If yes, it outputs $w$; otherwise, it outputs $\perp$.

We now define the notion of a robust sketch:

Definition 5. Given algorithms (SS, Rec) and random variables $\mathcal{W}=\left\{W_{0}, W_{1}\right.$, $\left.\ldots, W_{n}\right\}$ over metric space $(\mathcal{M}, d)$, consider the following game between an adversary $\mathcal{A}$ and a challenger: Let $w_{0}$ (resp., $w_{i}$ ) be the value assumed by $W_{0}$ (resp., $\left.W_{i}\right)$. The challenger computes pub $\leftarrow \mathrm{SS}\left(w_{0}\right)$ and gives pub to $\mathcal{A}$. Next, for $i=1, \ldots, n$, the adversary $\mathcal{A}$ outputs a "challenge" $\mathrm{pub}_{i} \neq \mathrm{pub}$ and is given $\operatorname{Rec}\left(w_{i}, \operatorname{pub}_{i}\right)$ in return. If there exists an $i$ such that $\operatorname{Rec}\left(w_{i}, \operatorname{pub}_{i}\right) \neq \perp$ we say that the adversary succeeds and this event is denoted by Succ.

We say that (SS, Rec) is an $\left(m, m^{\prime \prime}, n, \varepsilon, t\right)$-robust sketch over $(\mathcal{M}, d)$ if it is a well-formed $(m, \star, t)$-secure sketch and: (1) for all $t$-bounded distortion ensembles $\mathcal{W}$ with $H_{\infty}\left(W_{0}\right) \geq m$ and all adversaries $\mathcal{A}$ we have $\operatorname{Pr}[\mathrm{Succ}] \leq \varepsilon$; and (2) the average min-entropy of $W_{0}$, conditioned on the entire view of $\mathcal{A}$ throughout the above game, is at least $m^{\prime \prime} 2$

A simpler definition would be to consider only random variables $\left\{W_{0}, W_{1}\right\}$ and to have $\mathcal{A}$ only output a single value $\operatorname{pub}_{1} \neq$ pub. A standard hybrid argument would then imply the above definition with $\varepsilon$ increased by a multiplicative factor of $n$. We have chosen to work with the more general definition above as it potentially allows for a tighter concrete security analysis. Also, although the above definition allows all-powerful adversaries, we will consider adversaries whose queries to a random oracle are bounded (but which are otherwise computationally unbounded). We remark that for a truly unbounded adversary (i.e., where even the oracle queries - if any — are unbounded), it is necessarily the case that $m^{\prime \prime} \geq \log \frac{1}{\varepsilon}$ since at the last step of the game the adversary can guess $W_{n}$ with probability $2^{-m^{\prime \prime}}$ and thus succeed with probability $\varepsilon \geq 2^{-m^{\prime \prime}}$.

\subsection{Constructing a Generic Robust Sketch}

Let $H:\{0,1\}^{*} \rightarrow\{0,1\}^{k}$ be a hash function. We construct a robust sketch (SS, Rec) from any well-formed secure sketch $\left(\mathrm{SS}^{*}, \mathrm{Rec}^{*}\right)$ as follows:

$$
\begin{array}{l|l}
\frac{\mathrm{SS}(w)}{\operatorname{pub}^{*} \leftarrow \mathrm{SS}^{*}(w)} & \frac{\operatorname{Rec}\left(w, \operatorname{pub}=\left\langle\mathrm{pub}^{*}, h\right\rangle\right)}{w^{\prime}=\operatorname{Rec}^{*}\left(w, \mathrm{pub}^{*}\right)} \\
h=H\left(w, \mathrm{pub}^{*}\right) & \text { if } w^{\prime}=\perp \text { output } \perp \\
\text { return pub }=\left\langle\mathrm{pub}^{*}, h\right\rangle & \begin{array}{l}
\text { if } H\left(w^{\prime}, \text { pub* }\right) \neq h \text { output } \perp \\
\text { otherwise, output } w^{\prime}
\end{array}
\end{array}
$$

Theorem 1. If (SS*, Rec $\left.{ }^{*}\right)$ is a well-formed $\left(m, m^{\prime}, t\right)$-secure sketch over metric space $(\mathcal{M}, d)$ and $H:\{0,1\}^{*} \rightarrow\{0,1\}^{k}$ is a random oracle, then (SS, Rec) is an

\footnotetext{
${ }^{2}$ In particular, this implies that (SS, Rec) is an $\left(m, m^{\prime \prime}, t\right)$-secure sketch.
} 
$\left(m, m^{\prime \prime}, n, \varepsilon, t\right)$-robust sketch over $(\mathcal{M}, d)$ for any adversary making at most $q_{H}$ queries to $H$, where

$$
\begin{aligned}
\varepsilon & =\left(q_{H}^{2}+n\right) \cdot 2^{-k}+\left(3 q_{H}+2 n \cdot \mathrm{Vol}_{t}^{\mathcal{M}}\right) \cdot 2^{-m^{\prime}}, \\
m^{\prime \prime} & =-\log \varepsilon .
\end{aligned}
$$

When $k \geq m^{\prime}+\log q_{H}$ (which can be enforced in practice), the above simplifies to $\varepsilon \leq\left(4 q_{H}+2 n \cdot \mathrm{Vol}_{t}^{\mathcal{M}}\right) \cdot 2^{-m^{\prime}}$ and $m^{\prime \prime} \geq m^{\prime}-\log \left(4 q_{H}+2 n \cdot \mathrm{Vol}_{t}^{\mathcal{M}}\right)$.

Proof (Sketch). It is easy to see that (SS, Rec) is an $(m, \star, t)$-secure sketch and thus we only need to prove the latter two conditions of Definition 5. In order to provide intuition, the following proof is somewhat informal; however, the arguments given here can easily be formalized. Let pub $=\left\langle\right.$ pub $\left.^{*}, h\right\rangle$ denote the value output by SS in an execution of the game described in Definition 5. Note that if $\mathcal{A}$ ever outputs $\mathrm{pub}_{i}=\left\langle\mathrm{pub}_{i}^{*}, h_{i}\right\rangle$ with pub $_{i}^{*}=\mathrm{pub}^{*}$ then the response is always $\perp$, since then we must have $h_{i} \neq h$ and so Rec will output $\perp$. Thus, we simply assume that $\operatorname{pub}_{i}^{*} \neq$ pub* $^{*}$.

Fix a $t$-bounded distortion ensemble $\left\{W_{0}, W_{1}, \ldots, W_{n}\right\}$ with $H_{\infty}\left(W_{0}\right) \geq m$. For any output pub $_{i}=\left\langle\operatorname{pub}_{i}^{*}, h_{i}\right\rangle$ of $\mathcal{A}$, define the random variable $W_{i}^{\prime} \stackrel{\text { def }}{=}$ $\operatorname{Rec}^{*}\left(W_{i}, \operatorname{pub}_{i}^{*}\right)$. In order not to complicate notation, we define

$$
H_{\infty}\left(W_{i}^{\prime}\right) \stackrel{\text { def }}{=}-\log \left(\max _{x \in \mathcal{M}} \operatorname{Pr}\left[W_{i}^{\prime}=x\right]\right) ;
$$

i.e., we ignore the probability that $W_{i}^{\prime}=\perp$ since $\mathcal{A}$ does not succeed in this case. $\bar{H}_{\infty}\left(W_{i}^{\prime} \mid X\right)$ for a random variable $X$ is defined similarly. Let $w_{0}, w_{i}$, and $w_{i}^{\prime}$ denote the values taken by the random variables $W_{0}, W_{i}$, and $W_{i}^{\prime}$, respectively.

We classify the random oracle queries of $\mathcal{A}$ into two types: type 1 queries are those of the form $H\left(\cdot\right.$, pub* $\left.^{*}\right)$, and type 2 queries are all the others. Informally, type 1 queries represent attempts by $\mathcal{A}$ to learn the value of $w_{0}$; in particular, if $\mathcal{A}$ finds $w$ such that $H\left(w\right.$, pub $\left.^{*}\right)=h$ then it is "likely" that $w_{0}=w$. Type 2 queries represent attempts by $\mathcal{A}$ to determine an appropriate value for some $h_{i}$; i.e., if $\mathcal{A}$ "guesses" that $w_{i}^{\prime}=w$ for a particular choice of pub $_{i}^{*}$ then a "winning" strategy is for $\mathcal{A}$ to obtain $h_{i}=H\left(w, \operatorname{pub}_{i}^{*}\right)$ and output $\operatorname{pub}_{i}=\left\langle\operatorname{pub}_{i}^{*}, h_{i}\right\rangle$.

Without loss of generality, we assume that $\mathcal{A}$ makes all its type 1 queries first, then makes all its type 2 queries, and finishes by making its $n$ queries to the challenger (cf. Definition 5) in parallel (i.e., non-adaptively). The validity of the assumption on the ordering of the type 1 and type 2 queries follows essentially from the analysis that follows. The assumption that all queries to the random oracle are made before any queries to the challenger is justified by the observation that if $\operatorname{Rec}\left(W_{i}, \operatorname{pub}_{i}\right) \neq \perp$ then the adversary has already succeeded, in which case we can end the game, whereas the only remaining response $\operatorname{Rec}\left(W_{i}, \operatorname{pub}_{i}\right)=\perp$ can be simulated by the adversary itself. This also justifies why we may assume that the adversary's challenges are made in parallel.

Let $\mathcal{Q}_{1}$ (resp., $\mathcal{Q}_{2}$ ) be a random variable denoting the sequence of type 1 (resp., type 2) queries made by $\mathcal{A}$ and the corresponding responses, and let $q_{1}$ (resp., $q_{2}$ ) denote the value assumed by $Q_{1}$ (resp., $Q_{2}$ ). For some fixed value of 
pub, define $\gamma_{\text {pub }} \stackrel{\text { def }}{=} H_{\infty}\left(W_{0} \mid\right.$ pub $)$. Notice, since $\left(\mathrm{SS}^{*}, \mathrm{Rec}^{*}\right)$ is an $\left(m, m^{\prime}, t\right)$-secure sketch, we have $\operatorname{Exp}_{\text {pub }}\left[2^{-\gamma_{\text {pub }}}\right] \leq 2^{-m^{\prime}}$. Now, define $\gamma_{\text {pub }, q_{1}}^{\prime} \stackrel{\text { def }}{=} H_{\infty}\left(W_{0} \mid\right.$ pub, $\left.q_{1}\right)$, and let us call the value $q_{1}$ "bad" if $\gamma_{\text {pub }, q_{1}}^{\prime} \leq \gamma_{\text {pub }}-1$. We consider two cases: If $2^{\gamma_{\text {pub }}} \leq 2 q_{H}$ we will not have any guarantees, but using Markov's inequality we have $\operatorname{Pr}\left[2^{\gamma_{\text {pub }}} \leq 2 q_{H}\right]=\operatorname{Pr}\left[2^{-\gamma_{\text {pub }}} \geq 2^{-m^{\prime}} \cdot\left(2^{m^{\prime}} / 2 q_{H}\right)\right] \leq 2 q_{H} \cdot 2^{-m^{\prime}}$. Otherwise, if $2^{\gamma_{\text {pub }}}>2 q_{H}$, we observe that the type 1 queries of $\mathcal{A}$ may be viewed as guesses of $w_{0}$. In fact, it is easy to see that we only improve the success probability of $\mathcal{A}$ if in response to a type 1 query of the form $H\left(w\right.$, pub $\left.^{*}\right)$ we simply tell $\mathcal{A}$ whether $w_{0}=w$ or not 3 It is immediate that $\mathcal{A}$ learns the correct value of $w_{0}$ with probability at most $q_{H} \cdot 2^{-\gamma_{\text {pub }}}$. Moreover, when this does not happen, $\mathcal{A}$ has eliminated at most $q_{H} \leq 2^{\gamma_{\text {pub }}} / 2$ (out of at least $2^{\gamma_{\text {pub }}}$ ) possibilities for $w_{0}$, which means that $\gamma_{\text {pub }, q_{1}}^{\prime} \geq \gamma_{\text {pub }}-1$, or in other words that $q_{1}$ is "good". Therefore, the probability that $q_{1}$ is "bad" in this second case is at most $q_{H} \cdot 2^{-\gamma_{\text {pub }}}$.

Combining the above two arguments, we see that

$$
\begin{aligned}
\operatorname{Exp}_{\text {pub }}\left[\operatorname{Pr}\left[q_{1} \mathrm{bad}\right]\right] & \leq \operatorname{Pr}_{\text {pub }}\left[2^{\gamma_{\text {pub }}} \leq 2 q_{H}\right]+\operatorname{Exp}_{\text {pub }}\left[q_{H} \cdot 2^{-\gamma_{\text {pub }}}\right] \\
& \leq 2 q_{H} \cdot 2^{-m^{\prime}}+q_{H} \cdot 2^{-m^{\prime}}=3 q_{H} \cdot 2^{-m^{\prime}} .
\end{aligned}
$$

Next, define $\gamma_{\text {pub }, q_{1}}^{\prime \prime} \stackrel{\text { def }}{=} \min _{i}\left(H_{\infty}\left(W_{i}^{\prime} \mid\right.\right.$ pub, $\left.\left.q_{1}\right)\right)$. Recall that $\left\{W_{0}, W_{1}, \ldots\right\}$ is a $t$-bounded distortion ensemble which means $d\left(W_{0}, W_{i}\right) \leq t$. Furthermore, since $\left(\mathrm{SS}^{*}, \mathrm{Rec}^{*}\right)$ is well-formed, $\left\{W_{i}, W_{i}^{\prime}\right\}$ is also a $t$-bounded distortion ensembl 4 regardless of pub ${ }_{i}^{*}$, which means $d\left(W_{i}, W_{i}^{\prime}\right) \leq t$. Applying Lemma 2 on $\left\{W_{0}, W_{i}\right\}$ (noticing that pub contains pub*), followed by Lemma 1 on $\left\{W_{i}, W_{i}^{\prime}\right\}$, we have

$$
\gamma_{\text {pub }, q_{1}}^{\prime \prime} \geq \min _{i}\left(H_{\infty}\left(W_{i} \mid \text { pub }, q_{1}\right)\right)-\log \operatorname{Vol}_{t}^{\mathcal{M}} \geq \gamma_{\text {pub }, q_{1}}^{\prime}-\log \operatorname{Vol}_{t}^{\mathcal{M}} .
$$

We now consider the type 2 queries made by $\mathcal{A}$. Clearly, the answers to these queries do not affect the conditional min-entropies of $W_{i}^{\prime}$ (since these queries do not include pub*), so the best probability for the attacker to predict any of the $W_{i}^{\prime}$ is still given by $2^{-\gamma_{\text {pub } q_{1}}^{\prime \prime}}$, for fixed pub and $q_{1}$. Assume for a moment that there are no collisions in the outputs of any of the adversary's random oracle queries, and consider the adversary's $i^{\text {th }}$ query $\left\langle\right.$ pub $\left._{i}^{*}, h_{i}\right\rangle$ to the challenger. The probability that this query is "successful" is at most the probability that $\mathcal{A}$ asked a type 2 query of the form $H\left(w_{i}^{\prime}, \cdot\right)$ for the correct $w_{i}^{\prime}$ plus the probability that such a query was not asked, yet $\mathcal{A}$ nevertheless managed to predict the value $H\left(w_{i}^{\prime}\right.$, pub $\left._{i}^{*}\right)$. Clearly, the second case happens with probability at most $2^{-k}$. As for the first case, for any $h_{i}$ there is at most one $w$ for which $H(w, \cdot)=h_{i}$, since, by assumption, there are no collisions in these type 2 queries. Thus, the adversary succeeds on its $i^{\text {th }}$ query if this $w$ is equal to the correct value $w_{i}^{\prime}$. By what we just argued, the probability that this occurs is at most $2^{-\gamma_{\text {pub }, q_{1}}^{\prime \prime}}$, irrespective of pub* $i_{i}^{*}$.

${ }^{3}$ This has no effect when $H\left(w\right.$, pub $\left.^{*}\right) \neq h$ as then $\mathcal{A}$ learns anyway that $w \neq w_{0}$. The modification has a small (but positive) effect on the success probability of $\mathcal{A}$ when $H\left(w\right.$, pub $\left.^{*}\right)=h$ since this fact by itself does not definitively guarantee that $w=w_{0}$.

${ }^{4}$ This ignores the case when $W_{i}^{\prime}=\perp$; see the definition of $H_{\infty}\left(W_{i}^{\prime}\right)$ given earlier. 
Therefore, assuming no collisions in type 2 queries, the success probability of $\mathcal{A}$ in any one of its $n$ parallel queries is at most $n \cdot\left(2^{-\gamma_{\text {pub }, q_{1}}^{\prime \prime}}+2^{-k}\right)$. Furthermore, by the birthday bound the probability of a collision is at most $q_{H}^{2} / 2^{k}$. Therefore, conditioned on pub and $q_{1}$ and for the corresponding value of $\gamma_{\text {pub, } q_{1}}^{\prime \prime}$, we find that $\operatorname{Pr}\left[\right.$ Succ $\mid$ pub, $\left.q_{1}\right] \leq n \cdot 2^{-\gamma_{\text {pub }, q_{1}}^{\prime \prime}}+\left(q_{H}^{2}+n\right) \cdot 2^{-k}$.

To conclude, the adversary's overall probability of success is thus bounded by the expectation, over pub and $q_{1}$, of this previous quantity; that is:

$$
\begin{aligned}
& \operatorname{Pr}[\text { Succ }]=\operatorname{Exp}_{\text {pub }, q_{1}}\left[\operatorname{Pr}\left[\text { Succ } \mid \text { pub }, q_{1}\right]\right] \\
& \leq\left(q_{H}^{2}+n\right) \cdot 2^{-k} \\
& \quad+\operatorname{Exp}_{\text {pub }}\left[\operatorname{Pr}_{q_{1} \leftarrow \mathcal{Q}_{1}}\left[q_{1} \text { bad } \mid \text { pub }\right]+\sum_{q_{1} \text { good }} n \cdot 2^{-\gamma_{\text {pub }, q_{1}}^{\prime \prime} \cdot \operatorname{Pr}\left[Q_{1}=q_{1} \mid \text { pub }\right]}\right] .
\end{aligned}
$$

Using Equation 2, we see that $2^{-\gamma_{\text {pub }, q_{1}}^{\prime \prime}} \leq \mathrm{Vol}_{t}^{\mathcal{M}} \cdot 2^{-\gamma_{\text {pub }, q_{1}}^{\prime}}$. Moreover, for good $q_{1}$

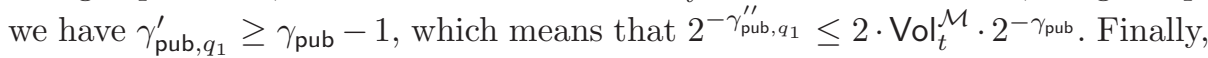
using Equation 1, we have $\operatorname{Exp}_{\text {pub }}\left[\operatorname{Pr}\left[q_{1}\right.\right.$ bad $\mid$ pub $\left.]\right] \leq 3 q_{H} \cdot 2^{-m^{\prime}}$. Combining all these, we successively derive:

$$
\begin{aligned}
& \operatorname{Pr}[\text { Succ }] \leq\left(q_{H}^{2}+n\right) \cdot 2^{-k}+3 q_{H} \cdot 2^{-m^{\prime}} \\
& +\operatorname{Exp}_{\text {pub }}\left[2 n \cdot \operatorname{Vol}_{t}^{\mathcal{M}} \cdot 2^{-\gamma_{\text {pub }}} \cdot \operatorname{Pr}_{q_{1} \leftarrow \mathcal{Q}_{1}}\left[q_{1} \text { good }\right]\right] \\
& \leq\left(q_{H}^{2}+n\right) \cdot 2^{-k}+3 q_{H} \cdot 2^{-m^{\prime}}+2 n \cdot \operatorname{Vol}_{t}^{\mathcal{M}} \cdot \operatorname{Exp}_{\mathrm{pub}}\left[2^{-\gamma_{\mathrm{pub}}}\right] \\
& \leq\left(q_{H}^{2}+n\right) \cdot 2^{-k}+\left(3 q_{H}+2 n \cdot \operatorname{Vol}_{t}^{\mathcal{M}}\right) \cdot 2^{-m^{\prime}}=\varepsilon .
\end{aligned}
$$

As for the claimed value of $m^{\prime \prime}$, we omit the details since they follow almost the same argument. As above, assuming that $q_{1}$ is good, that no collisions occur in type 2 queries, and that the adversary does not manage to guess any of the values $H\left(w_{i}^{\prime}\right.$, pub $\left.i_{i}^{*}\right)$, the conditional min-entropy of $W_{0}$ is at least $\gamma_{\text {pub, } q_{1}}^{\prime}-\log (n$. $\left.\operatorname{Vol}_{t}^{\mathcal{M}}\right) \geq \gamma_{\text {pub }}-1-\log \left(n \cdot \operatorname{Vol}_{t}^{\mathcal{M}}\right)$. On the other hand, all these bad events leading to a possibly smaller min-entropy of $W_{0}$ happen with (expected) probability (over pub) at most $\left(q_{H}^{2}+n\right) \cdot 2^{-k}+3 q_{H} \cdot 2^{-m^{\prime}}$. From this, it is easy to see that if View represents the adversary's view in the experiment, then

$$
\begin{aligned}
\bar{H}_{\infty}\left(W_{0} \mid \text { View }\right) \geq & -\log \left(\left(q_{H}^{2}+n\right) \cdot 2^{-k}+3 q_{H} \cdot 2^{-m^{\prime}}\right. \\
& \left.+\operatorname{Exp}_{\text {pub }}\left[2 n \cdot \operatorname{Vol}_{t}^{\mathcal{M}} \cdot 2^{-\gamma_{\text {pub }}}\right]\right) \\
\geq & -\log \left(\left(q_{H}^{2}+n\right) \cdot 2^{-k}+\left(3 q_{H}+2 n \cdot \operatorname{Vol}_{t}^{\mathcal{M}}\right) \cdot 2^{-m^{\prime}}\right)=m^{\prime \prime} .
\end{aligned}
$$

We remark that the above proof uses only a non-programmable random oracle.

The bound on $\varepsilon$ that we derive in the above proof has an intuitive interpretation. The sub-expression $\left(q_{H}+n \cdot \mathrm{Vol}_{t}^{\mathcal{M}}\right) \cdot 2^{-m^{\prime}}$ that appears (up to a small 
constant factor due to the analysis) can be viewed as the probability that the adversary "gets information" about the point $w_{0}$ : The contribution $q_{H} \cdot 2^{-m^{\prime}}$ is due to the type 1 oracle queries where, for each of at most $q_{H}$ queries, the adversary "hits" the correct value of $w_{0}$ with probability $2^{-m^{\prime}}$. Then, each of the adversary's $n$ challenges cover no more than $\mathrm{Vol}_{t}^{\mathcal{M}}$ candidates for $w_{0}$, since each such query eliminates at most one value for $w_{i}^{\prime}$ (unless collisions in type 2 queries occur), which in turn eliminates up to $\mathrm{Vol}_{t}^{\mathcal{M}}$ candidates for $w_{i}$, each of which can only eliminate one candidate $\operatorname{Rec}\left(w_{i}, \operatorname{pub}^{*}\right)$ for $w_{0}$. Besides the above, the other contributions to $\varepsilon$ are due to the probability of collisions in the random oracle, plus a small term to account for the possibility that the adversary can guess the output of the random oracle at an unqueried point.

In practice, one can set $k$ large enough so that $\max \left(q_{H}, n \cdot \mathrm{Vol}_{t}^{\mathcal{M}}\right)$ is the dominant factor determining the amount of the additional "loss" incurred as compared to regular "non-robust" sketches.

\subsection{From Robust Sketches to Robust Fuzzy Extractors}

In a manner exactly analogous to the above, we may define the notion of a robust fuzzy extractor. We include the definition here since we refer to it in the next subsection:

Definition 6. Given algorithms (Ext, Rec) and random variables $\mathcal{W}=\left\{W_{0}\right.$, $\left.W_{1}, \ldots, W_{n}\right\}$ over a metric space $(\mathcal{M}, d)$, consider the following game between an adversary $\mathcal{A}$ and a challenger: Let $w_{0}$ (resp., $w_{i}$ ) be the value assumed by $W_{0}$ (resp., $\left.W_{i}\right)$. The challenger computes $(R, \mathrm{pub}) \leftarrow \operatorname{Ext}\left(w_{0}\right)$ and gives pub to A. Next, for $i=1, \ldots, n$, the adversary $\mathcal{A}$ outputs $\mathrm{pub}_{i} \neq$ pub and is given $\operatorname{Rec}\left(w_{i}, \operatorname{pub}_{i}\right)$ in return. If there exists an $i$ such that $\operatorname{Rec}\left(w_{i}, \operatorname{pub}_{i}\right) \neq \perp$, we say the adversary succeeds and this event is denoted by Succ.

We say (Ext, Rec) is an $(m, \ell, n, \varepsilon, t, \delta)$-robust fuzzy extractor over $(\mathcal{M}, d)$ if the following hold for all t-bounded distortion ensembles $\mathcal{W}$ with $H_{\infty}\left(W_{0}\right) \geq m$ :

(Robustness) For all adversaries $\mathcal{A}$, it holds that $\operatorname{Pr}[\mathrm{Succ}] \leq \varepsilon$.

(Security) Let View denote the entire view of $\mathcal{A}$ at the conclusion of the above game. Then, $\mathbf{S D}\left(\langle R, \mathrm{View}\rangle,\left\langle U_{\ell}, \mathrm{View}\right\rangle\right) \leq \delta$.

(Error-tolerance) For all $w^{\prime}$ with $d\left(w_{0}, w^{\prime}\right) \leq t$, we have $\operatorname{Rec}\left(w^{\prime}, \operatorname{pub}\right)=R$. $\diamond$

By applying a pairwise-independent hash function (i.e., a strong extractor) almost exactly as in [9-Lemma 3.1], we can convert any robust sketch to a robust fuzzy extractor in the standard model (losing, as there, $2 \log \delta^{-1}$ bits of entropy). A subtlety is that we need to "bind" the hash function key to the sketch itself. We do this using a primitive we call a labeled robust sketch which, in a nutshell, defines a family of robust sketches indexed by a label. (For example, in the specific construction of robust sketches from the previous section labels can be incorporated by including the label as the input to the hash function which is modeled as a random oracle.) By using the key to the hash function as a label, we can bind the key to the sketch as needed. Details will appear in the full version. 
We remark that if we are content to assume a random oracle $G$ (as we anyway only know how to construct robust sketches in the random oracle model), we can trivially "extract" from a random variable $w$ by computing $G(w)$. This has the advantage of not losing $2 \log \delta^{-1}$ bits of entropy when extracting. In this case, we achieve $\delta \leq q_{G} \cdot 2^{-m^{\prime \prime}}$, where $q_{G}$ is the number of queries to $G$ and $m^{\prime \prime}$ is as in Theorem 1 .

\subsection{Application to Secure Authentication}

The application of a robust fuzzy extractor to achieve mutual authentication or authenticated key exchange over an insecure channel is immediate. Given any secure protocol $\Pi$ (say, for authenticated key exchange) based on a uniformlydistributed shared key of length $\ell$, any $(m, \ell, n, \varepsilon, t, \delta)$-robust fuzzy extractor (Ext, Rec), and any source $W_{0}$ with $H_{\infty}\left(W_{0}\right) \geq m$, consider the protocol $\Pi^{\prime}$ constructed as follows:

Initialization. The user samples $w_{0}$ according to $W_{0}$ (i.e., takes a scan of his biometric data) and computes $(R, \mathrm{pub}) \leftarrow \operatorname{Ext}\left(w_{0}\right)$. The user registers $(R$, pub) at the server.

Protocol execution. The $i^{\text {th }}$ time the user wants to run the protocol, the user will sample $w_{i}$ according to some distribution $W_{i}$ (i.e., the user re-scans his biometric data). The server sends pub to the user, who then computes $\hat{R}=\operatorname{Ext}\left(w_{i}\right.$, pub). If $\hat{R}=\perp$, the user immediately aborts. Otherwise, the server and user execute protocol $\Pi$, with the server and the user respectively using the keys $R$ and $\hat{R}$.

Assume that $\mathcal{W}=\left\{W_{0}, W_{1}, \ldots\right\}$ is a $t$-bounded distortion ensemble. Correctness of the above protocol is easily seen to hold: if the user obtains the correct value of pub from the server then, because $d\left(w_{0}, w_{i}\right) \leq t$, the user will recover $\hat{R}=R$ and thus both user and server will end up using the same key $R$ in the underlying protocol $\Pi$. The security of $\Pi^{\prime}$ with respect to the definitions of [3, 1, which consider an active adversary who may control all messages sent between the user and the server, follows from the following observations:

- If the adversary forwards pub $\neq$ pub to at most $n$ different user-instances, these instances will all abort immediately (without running $\Pi$ ) except with probability at most $\varepsilon$. Thus, except with this probability, the adversary is limited to forwarding the correct value of pub.

- When the adversary forwards pub unchanged, the user and server run an execution of $\Pi$ using a key $R$ which is within statistical difference $\delta$ from a uniformly distributed $\ell$-bit key. Note that this is true even when conditioned on the view of the adversary in sessions when it does not forward pub unchanged (cf. Definition 6). Thus, assuming $\Pi$ is secure, the adversary will not succeed in "breaking" $\Pi^{\prime}$ in this case either.

In terms of concrete security, if the security of $\Pi$ against an adversary who executes at most $n$ sessions with the user and the server is $\varepsilon_{\Pi}$, then the security 
of $\Pi^{\prime}$ is $\varepsilon+\delta+\varepsilon_{\Pi}$. A formal proof following the above intuition is straightforward, and will appear in the full version of this work.

\section{Improved Solution Tailored for Mutual Authentication}

As discussed in the introduction, the robust sketches and fuzzy extractors described in the previous section provide a general mechanism for dealing with adversarial modification of the public value pub. In particular, taking any protocol based on the secure sketches or fuzzy extractors of [9] which is secure when the public value is assumed not to be tampered with, and plugging in a robust sketch or fuzzy extractor, yields a protocol secure against an adversary who may either modify the contents of the server - as in the case where the server itself is malicious - or else modify the value of pub when it is sent to the user.

For specific problems of interest, however, it remains important to explore solutions which might improve upon the general-purpose solution described above. In this section, we show that for the case of mutual authentication and/or authenticated key exchange an improved solution is indeed possible. As compared to the generic solution based on robust fuzzy extractors (cf. Section [3.3), the solution described here has the advantages that: (1) it is provably secure in the standard model; and (2) it can achieve improved bounds on the "effective entropy loss". We provide an overview of our solution now.

Given the proof of Theorem 1, the intuition behind our current solution is actually quite straightforward. As in that proof, let $\mathcal{W}=\left\{W_{0}, \ldots\right\}$ be a sequence of random variables where $W_{0}$ represents the initial recorded value of the user's biometric data and $W_{i}$ denotes the $i^{\text {th }}$ scanned value of the biometric data. Given a well-formed secure sketch $\left(\mathrm{SS}^{*}, \mathrm{Rec}^{*}\right)$ and a value $\mathrm{pub}_{i}^{*} \neq \mathrm{pub}^{*}=\mathrm{SS}^{*}\left(W_{0}\right)$ chosen by the adversary, let $W_{i}^{\prime} \stackrel{\text { def }}{=} \operatorname{Rec}\left(W_{i}, \operatorname{pub}_{i}^{*}\right)$ and define the min-entropy of $W_{i}^{\prime}$ as in the proof of Theorem 1] At a high level, Theorem 1 follows from the observations that: (1) the average min-entropy of $W_{i}^{\prime}$ is "high" for any value pub $_{i}^{*}$; and (2) since the adversary succeeds only if it can also output a value $h_{i}=H\left(W_{i}^{\prime}, \operatorname{pub}_{i}^{*}\right)$, where $H$ is a random oracle, the adversary is essentially unable to succeed with probability better than $2^{-H_{\infty}\left(W_{i}^{\prime}\right)}$ in the $i^{\text {th }}$ iteration. Crucial to the proof also is the fact that, except with "small" probability, the value $h=H\left(W_{0}\right.$, pub*) does not reduce the entropy of $W_{0}$ "very much" (again using the fact that $H$ is a random oracle).

The above suggests that another way to ensure that the adversary does not succeed with probability better than $2^{-H_{\infty}\left(W_{i}^{\prime}\right)}$ in any given iteration would be to have the user run an "equality test" using its recovered value $W_{i}^{\prime}$. If this equality test is "secure" (in some appropriate sense we have not yet defined) then the adversary will effectively be reduced to simply guessing the value of $W_{i}^{\prime}$, and hence its success probability in that iteration will be as claimed. Since we have already noted that the average min-entropy of $W_{i}^{\prime}$ is "high" when any well-formed secure sketch is used (regardless of the value pub* chosen by the adversary), this will be sufficient to ensure security of the protocol overall. 
Thinking about what notion of security this "equality test" should satisfy, one realizes that it must be secure for arbitrary distributions on the user's secret value, and not just uniform ones. Also, the protocol must ensure that each interaction by the adversary corresponds to a guess of (at most) one possible value for $W_{i}^{\prime}$. Finally, since the protocol is meant to be run over an insecure network, it must be "non-malleable" in some sense so that the adversary cannot execute a man-in-the-middle attack when the user and server are both executing the protocol. Finally, the adversary should not gain any information about the user's true secret $W_{0}$ (at least in a computational sense) after passively eavesdropping on multiple executions of the protocol. With the problem laid out in this way, it becomes clear that one possibility is to use a password-only authenticated key exchange (PAK) protocol [4, 1,6] as the underlying "equality test".

Although the above intuition is appealing, we remark that a number of subtleties arise when trying to apply this idea to obtain a provably secure solution. In particular, we will require the PAK protocol to satisfy a slightly stronger definition of security than that usually considered for PAK (cf. [1, 6, 12]); informally, the PAK protocol should remain "secure" even when: (1) the adversary can dynamically add clients to the system, with (unique) identities chosen by the adversary; (2) the adversary can specify non-uniform and dependent password distributions for these clients; and (3) the adversary can specify such distributions adaptively at the time the client is added to the system. Luckily, it is not difficult to verify that at least some existing protocols (e.g., [1, 17, 18, 11, 16]) satisfy a definition of this sort 5 (Interestingly, the recent definition of [7] seems to imply the above properties.) Due to lack of space, the formal definition of security required for our application is deferred to the full version.

\subsection{A Direct Construction}

With the above in mind, we now describe our construction. Let $\Pi$ be a PAK protocol and let (SS, Rec) be a well-formed secure sketch. Construct a modified protocol $\Pi^{\prime}$ as follows:

Initialization. User $U$ samples $w_{0}$ according to $W_{0}$ (i.e., takes a scan of his biometric data) and computes pub $\leftarrow \mathrm{SS}\left(w_{0}\right)$. The user registers $\left(w_{0}\right.$, pub) at the server $S$.

Protocol execution (server). The server sends pub to the user. It then executes protocol $\Pi$ using the following parameters: it sets its own "identity" (within $\Pi$ ) to be $S \|$ pub, its "partner identity" to be pid $=U \|$ pub, and the "password" to be $w_{0}$.

Protocol execution (user). The $i^{\text {th }}$ time the user executes the protocol, the user first samples $w_{i}$ according to distribution $W_{i}$ (i.e., the user re-scans his biometric data). The user also obtains a value pub' in the initial message it

${ }^{5}$ In fact, it is already stated explicitly in 17, 11, that the given protocols remain secure even under conditions 1 and 2 , and it is not hard to see that they remain secure under condition 3 as well. 
receives, and computes $w^{\prime}=\operatorname{Rec}\left(w_{i}\right.$, pub' $)$. If $w^{\prime}=\perp$ then the user simply aborts. Otherwise, the user executes protocol $\Pi$, setting its own "identity"

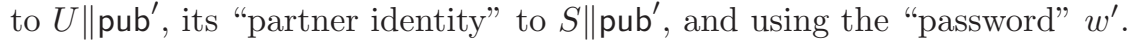

It is easy to see that correctness holds, since if the user and the server interact without any interference from the adversary then: (1) the identity used by the server is equal to the partner ID of the user; (2) the identity of the user is the same as the partner ID of the server; and (3) the passwords $w_{0}$ and $w^{\prime}$ are identical. Before discussing the security of this protocol, we need to introduce a slight restriction of the notion of a $t$-bounded distortion ensemble in which the various random variables in the ensemble are (efficiently) computable:

Definition 7. Let $(\mathcal{M}, d)$ be a metric space. An explicitly computable $t$-bounded distortion ensemble is a sequence of boolean circuits $\mathcal{W}=\left\{W_{0}, \ldots\right\}$ and a parameter $\ell$ such that, for all $i$, the circuit $W_{i}$ computes a function from $\{0,1\}^{\ell}$ to $\mathcal{M}$ and, furthermore, for all $r \in\{0,1\}^{\ell}$ we have $d\left(W_{0}(r), W_{i}(r)\right) \leq t$.

In our application, $\mathcal{W}$ will be output by a PPT adversary, ensuring both that the ensemble contains only a polynomial number of circuits and that each such circuit is of polynomial size (and hence may be evaluated efficiently). We remark that it is not necessary for our proof that it be possible to efficiently verify whether a given $\mathcal{W}$ satisfies the " $t$-bounded" property or whether the min-entropy of $W_{0}$ is as claimed, although the security guarantee stated below only holds if $\mathcal{W}$ does indeed satisfy these properties 6 With the above in mind, we now state the security achieved by our protocol:

Theorem 2. Let $\Pi$ be a secure PAK protocol (with respect to the definition sketched earlier) and let $\mathcal{A}$ be a PPT adversary. If (SS, Rec) is a well-formed $\left(m, m^{\prime}, t\right)$-secure sketch over a metric space $(\mathcal{M}, d)$, and $\mathcal{W}=\left\{W_{0}, \ldots\right\}$ is an explicitly-computable $t$-bounded distortion ensemble (output adaptively by $\mathcal{A}$ ) with $H_{\infty}\left(W_{0}\right) \geq m$, then the success probability of $\mathcal{A}$ in attacking protocol $\Pi^{\prime}$ is at most $q_{s} \cdot 2^{-m^{\prime \prime}}+\operatorname{negl}(\kappa)$, where $q_{s}$ represents the number of sessions in which the adversary attempts to impersonate one of the parties, and $m^{\prime \prime}=m^{\prime}-\log \operatorname{Vol}_{t}^{\mathcal{M}}$.

Due to space limitations, the proof is deferred to the full version.

Specific instantiations. As noted earlier, a number of PAK protocols satisfying the required definition of security are known. If one is content to work in the random oracle model then the protocol of [1] may be used (note that this still represents an improvement over the solution based on robust fuzzy extractors since the "effective key size" will be larger, as we discuss in the next paragraph). To obtain a solution in the standard model which is only slightly less efficient, the

\footnotetext{
${ }^{6}$ As to whether the adversary can be "trusted" to output a $\mathcal{W}$ satisfying these properties, recall that $\mathcal{W}$ anyway is meant to model naturally-occurring errors. Clearly, if a real-world adversary has the ability to, e.g., introduce arbitrarily-large errors then only weaker security guarantees can be expected to hold.
} 
PAK protocols of [17, 11, 16] could be used 7 Note that although these protocols were designed for use with "short" passwords, they can be easily modified to handle "large" passwords without much loss of efficiency; we discuss this further in the full version.

\subsection{Comparing Our Two Solutions}

It is somewhat difficult to compare the security offered by our two solutions (i.e., the one based on robust fuzzy extractors and the one described in this section) since an exact comparison depends on a number of assumptions and design decisions. As we already observed, the main advantage of the solution described in this section is that it does not rely on random oracles. On the other hand, the solution based on robust fuzzy extractors is simpler and more efficient.

The solution presented in this section does not require any randomness extraction, and it therefore "saves" $2 \log \delta^{-1}$ bits of entropy as compared with solutions that apply standard randomness extractors to the recovered biometric data. Since a likely value in practice is $\delta \leq 2^{-64}$, this results in a potential savings of at least 128 bits of entropy. When the entropy of the original biometric data is "large", however, we notice that (1) as mentioned already in the previous section, we may use a random oracle as our randomness extractor and thereby avoid the loss of $2 \log \delta^{-1}$ bits of entropy; and (2) our two approaches can be combined, and one can use a PAK protocol with any robust sketch. If this is done then additional extraction is not required, and so we again avoid losing $2 \log \delta^{-1}$ bits of entropy.

On the other hand, the solution of the present section offers a clear advantage when the entropy of the original biometric data is "small". Although in this case the adversary can succeed by an exhaustive, on-line "dictionary" attack, the security of our second solution implies that this is the best an adversary can do. In contrast, our solution based on robust sketches would not be appropriate in this case since the adversary could determine the user's secret biometric data using off-line queries to the random oracle (cf. the factor proportional to $q_{H} \cdot 2^{-m^{\prime}}$ in Theorem 1).

\section{References}

1. M. Bellare, D. Pointcheval, and P. Rogaway. Authenticated Key Exchange Secure Against Dictionary Attacks. Adv. in Cryptology - Eurocrypt 2000, LNCS vol. 1807, Springer-Verlag, pp. 139-155, 2000.

2. M. Bellare and P. Rogaway. Random Oracles are Practical: A Paradigm for Designing Efficient Protocols. ACM CCS 1993, ACM Press, 1993.

3. M. Bellare and P. Rogaway. Entity Authentication and Key Distribution. Adv. in Cryptology - Crypto 1993, LNCS vol. 773, Springer-Verlag, pp. 232-249, 1993.

\footnotetext{
${ }^{7}$ Although these protocols require public parameters, such parameters can be "hard coded" into the implementation of the protocol and are fixed for all users of the system; thus, users are not required to remember or store these values. The difference is akin to the difference between PAK protocols in a "hybrid" PKI model (where clients store their server's public key) and PAK protocols (including [17, 11, 16]) in which clients need only remember a short password.
} 
4. S. Bellovin and M. Merritt. Encrypted Key Exchange: Password-Based Protocols Secure Against Dictionary Attacks. IEEE Symposium on Research in Security and Privacy, IEEE, pp. 72-84, 1992.

5. X. Boyen. Reusable Cryptographic Fuzzy Extractors. ACM CCS 2004, ACM Press, pp. 82-91, 2004.

6. V. Boyko, P. MacKenzie, and S. Patel. Provably-Secure Password-Authenticated Key Exchange Using Diffie-Hellman. Adv. in Cryptology - Eurocrypt 2000, LNCS vol. 1807, Springer-Verlag, pp. 156-171, 2000.

7. R. Canetti, S. Halevi, J. Katz, Y. Lindell, and P. MacKenzie. Universally Composable Password-Based Key Exchange. Eurocrypt 2005 (these proceedings).

8. G. Davida, Y. Frankel, and B. Matt. On Enabling Secure Applications Through Off-Line Biometric Identification. IEEE Security and Privacy '98.

9. Y. Dodis, L. Reyzin, and A. Smith. Fuzzy Extractors: How to Generate Strong Keys from Biometrics and Other Noisy Data. Adv. in Cryptology - Eurocrypt 2004, LNCS vol. 3027, Springer-Verlag, pp. 523-540, 2004.

10. N. Frykholm and A. Juels. Error-Tolerant Password Recovery. ACM CCS 2001.

11. R. Gennaro and Y. Lindell. A Framework for Password-Based Authenticated Key Exchange. Adv. in Cryptology - Eurocrypt 2003, LNCS vol. 2656, Springer-Verlag, pp. 524-543, 2003.

12. O. Goldreich and Y. Lindell. Session-Key Generation Using Human Passwords Only. Adv. in Cryptology - Crypto 2001, LNCS vol. 2139, Springer-Verlag, pp. 408-432, 2001.

13. A. Juels. Fuzzy Commitment. Slides from a presentation at DIMACS, 2004. Available at http://dimacs.rutgers.edu/Workshops/Practice/slides/juels.ppt

14. A. Juels and M. Sudan. A Fuzzy Vault Scheme. IEEE Intl. Symp. on Info. Theory, 2002.

15. A. Juels and M. Wattenberg. A Fuzzy Commitment Scheme. ACM CCS 1999, ACM Press, 1999.

16. J. Katz, P. MacKenzie, G. Taban, and V. Gligor. Two-Server Password-Only Authenticated Key Exchange. Manuscript, Jan. 2005.

17. J. Katz, R. Ostrovsky, and M. Yung. Efficient Password-Authenticated Key Exchange Using Human-Memorable Passwords. Adv. in Cryptology - Eurocrypt 2001, LNCS vol. 2045, Springer-Verlag, pp. 475-494, 2001.

18. J. Katz, R. Ostrovsky, and M. Yung. Forward Secrecy in Password-Only KeyExchange Protocols. Security in Communication Networks: SCN 2002, LNCS vol. 2576, Springer-Verlag, pp. 29-44, 2002.

19. F. Monrose, M. Reiter, and S. Wetzel. Password Hardening Based on Keystroke Dynamics. ACM CCS 1999, ACM Press, 1999.

20. N. Nisan and A. Ta-Shma. Extracting Randomness: A Survey and New Constructions. J. Computer and System Sciences 58(1): 148-173, 1999.

21. P. Tuyls and J. Goseling. Capacity and Examples of Template-Protecting Biometric Authentication Systems. Biometric Authentication Workshop, 2004.

22. E. Verbitskiy, P. Tuyls, D. Denteneer, and J.-P. Linnartz. Reliable Biometric Authentication with Privacy Protection. 24th Benelux Symp. on Info. Theory, 2003. 